\title{
Interleukin-6 and C-reactive protein/ albumin ratio as predictors of COVID-19 severity and mortality
}

\author{
Mohamed El-Shabrawy ${ }^{1 *}$ D, Maha E. Alsadik', Maher El-Shafei ${ }^{2}$, Ahmed A. Abdelmoaty ${ }^{3}$, Ahmed S. Alazzouni ${ }^{4}$, \\ Marwa M. Esawy ${ }^{5}$ and Marwa A. Shabana ${ }^{5}$
}

\begin{abstract}
Background: Coronavirus disease 2019 (COVID-19) was announced in early December 2019. The pandemic situation is declared. This study aimed to evaluate the role of biomarkers in estimating the severity and predicting the prognosis of COVID-19.

Results: A total of 116 confirmed patients were included in this study. The patients were evaluated clinically. The disease severity was assessed. The measured and calculated laboratory tests were done. The primary outcome is the 30-day mortality. Patients were assigned to the severe (14.7\%) and non-severe (85.3\%) groups. At IL-6 level of 32.3 $\mathrm{pg} / \mathrm{mL}$ (the highest Youden's index $=0.77$ ), IL-6 can differentiate severe from non-severe patients with $82.4 \%$ sensitivity and $94.4 \%$ specificity. IL-6 can predict the severity [odds ratio of $87.7(95 \% \mathrm{Cl}=18.9-408.2)(P<0.0001)]$. After adjustment to the significant clinical and laboratory parameters, IL-6 had an adjusted odds ratio of 30.8 (95\% $\mathrm{Cl}=1.1-728.3)(P=0.046)$. A high CRP/albumin ratio of $>11.4$ was associated with COVID-19 mortality [hazard ratio $=59.9(95 \% \mathrm{Cl}=7.4-488.3)(P<0.0001)]$. High CRP/albumin ratio had an adjusted hazard ratio of $26.5(95 \% \mathrm{Cl}=$ 2.6-270.7) after adjustment of age and presence of co-morbidities $(P=0.006)$.

Conclusion: IL-6 level could effectively discriminate COVID-19 severity. CRP/albumin ratio was an independent risk factor for 30-day mortality rate in patients with COVID-19. IL-6 and CRP/albumin ratio seem to be valuable biomarkers in evaluating the severity and prognosis of COVID-19, respectively.
\end{abstract}

Keywords: COVID-19, C-reactive protein/albumin ratio, Interleukin-6, Mortality, Severity

\section{Background}

Coronavirus disease 2019 (COVID-19) was announced in early December 2019. By genome sequencing, the virus was recognized [1]. From Wuhan City, the virus spread globally. The pandemic situation is declared by the World Health Organization [2]. In Egypt, the first case was detected on 14 February 2020, for a foreigner. After nearly 2 weeks, the Egyptian cases were detected [3].

\footnotetext{
* Correspondence: shabrawy_m@yahoo.com

${ }^{1}$ Chest Medicine Department, Faculty of Human Medicine, Zagazig University, Zagazig, Egypt

Full list of author information is available at the end of the article
}

COVID-19 clarified as a highly transmissible disease. The clinical features varied with disease severity. Most COVID-19 patients have non-severe manifestations and show a good prognosis. However, patients with severe disease may progress to pulmonary dysfunction, multiple organ dysfunction, and death [4]. COVID-19 related to a considerable mortality rate in older patients and cases had other morbidities [5].

In other coronaviruses, studies suggested that the inflammatory storm is a common finding [6]. Similarly, increases in the inflammatory markers like interleukin-6 (IL-6) and C-reactive protein (CRP) were described in COVID-19 [7]. Albumin levels decreased in the inflammatory conditions; reduced levels were confirmed in

\section{Springer Open}

(อ) The Author(s). 2021 Open Access This article is licensed under a Creative Commons Attribution 4.0 International License, which permits use, sharing, adaptation, distribution and reproduction in any medium or format, as long as you give appropriate credit to the original author(s) and the source, provide a link to the Creative Commons licence, and indicate if changes were made. The images or other third party material in this article are included in the article's Creative Commons licence, unless indicated otherwise in a credit line to the material. If material is not included in the article's Creative Commons licence and your intended use is not permitted by statutory regulation or exceeds the permitted use, you will need to obtain permission directly from the copyright holder. To view a copy of this licence, visit http://creativecommons.org/licenses/by/4.0/. 
severe COVID-19 patients [8]. Hypoalbuminemia and high CRP/albumin ratio were previously linked to the mortality of various clinical conditions as critically ill patients [9].

To avoid the unnecessary or inappropriate utilization of the healthcare resources, early prediction of the severity of COVID-19 will be helpful. Severity prediction will also improve the prognosis by reducing the mortality rate [10]. Thus, this study aimed to evaluate the role of inflammatory markers in estimating the severity and predicting the prognosis of COVID-19. This study hypothesized that elevated values of IL-6, CRP, and CRP/ albumin ratio at the time of COVID-19 diagnosis are associated with COVID-19 severity and mortality.

\section{Methods}

\section{Study design}

In June 2020, a cohort study was carried out on patients from Zagazig University Hospitals. The subjects' participation was confirmed by signing the consent form either by the patient or the first degree relatives. The protocol of this study was affirmed by the Institutional Review Board of the Faculty of Human Medicine, Zagazig University (approval number: 6250). The patients were evaluated by full history and clinical examinations. The disease severity was assessed. At the time of diagnosis, measured and calculated laboratory tests were done. The primary outcome was 30 -day mortality.

\section{Subjects}

The Epi Info program 6 (Atlanta, Ga, USA) was the utilized program for sample size estimation. The percentage of exposure and the outcome of either exposed or non-exposed groups were calculated following the study of Liu et al. [11] with $95 \%$ statistical power and $95 \%$ confidence limit. A total of 116 consecutive confirmed patients were included. The diagnosis and severity classification criteria were performed as stated by the management protocol of COVID-19 released by the Egyptian Ministry of Health and Population (2020). All included patients were diagnosed as COVID-19 positive ones based on the presence of viral nucleic acid in the nasopharyngeal swab. By disease severity, patients were subgrouped. The patient considered as severe one if at least a single criterion of these criteria was present [increased respiration rate/minute $>30$ or reduced oxygen saturation at room air $<92 \%$ or decreased partial pressure of arterial oxygen to fraction of inspired oxygen ratio $<300$ $\mathrm{mmHg}$ or a chest lesion $>50 \%$ or a rapid advancement of chest damage within 24-48 h]. The critically ill patients were considered if their respiration rate/minute is $>30$ or have reduced oxygen saturation $<92 \%$ or have decreased partial pressure of arterial oxygen to fraction of inspired oxygen ratio < $200 \mathrm{mmHg}$; despite oxygen therapy. Both severe and critically ill cases were included in the severe group. The patients were divided into two groups, including 99 patients in the non-severe group and 17 cases in the severe one (Fig. 1).

\section{Samples}

In $\mathrm{BD}^{\circ}$ Plus Plastic Serum vacutainer, blood samples were obtained. The plain vacutainers were allowed to clot for $30 \mathrm{~min}$ after collection. After this period, samples were centrifuged at $1200 \times g$ for $10 \mathrm{~min}$ to separate serum. The analysis of CRP and albumin was performed immediately. The remaining serum was transferred in $1.5-\mathrm{mL}$ sterile microcentrifuge tubes and stored at $-80^{\circ} \mathrm{C}$ until IL-6 analysis.

\section{Methods}

CRP and albumin were quantified using the CRP and albumin LR kits, respectively (Gesan Production Kit, Campobello di Mazara, Trapani, Italy). These biochemical tests were performed according to the manufacturer's steps on the Microlab 300 (ELITech Group, France). IL-6 was quantified by Human IL-6 ELISA Kit (Bioassay Technology Laboratory, Shanghai, China) [Catalog No.: E0090Hu]. The assay steps were done according to the manufacturer's procedure. The readings were done using the BioTek ELx800 Absorbance Microplate Readers (Bio-Tek Instruments, USA). The IL-6 levels were expressed in $\mathrm{pg} / \mathrm{mL}$. The precision coefficients of this IL- 6 kit were an intra-assay CV of less than $10 \%$ and an inter-assay CV of less than $12 \%$.

\section{Calculations}

The calculated ratio was estimated using the following equation: "CRP/Albumin ratio $=\mathrm{CRP}$ level/Albumin level."

\section{Statistical analysis}

Data were checked by the Shapiro-Wilk test, a nonparametrically distribution was found. Mann-Whitney $U$ test and chi-square test were used for two groups' comparison. The performance of the laboratory test was evaluated by the receiver operator characteristic (ROC) curve. The optimal cutoff point was established by the highest Youden's index. The Logistic Regression Analysis was utilized to clarify the predictors by measuring the odds ratio (OR) and its 95\% confidence interval (CI). The outcome evaluation was performed by KaplanMeier survival analysis, log-rank tests, and the Coxregression analysis. The accepted statistically significant value was a $P$ value of less than 0.05. SPSS 16.0 (SPSS Inc., Chicago, IL, USA) was utilized. 


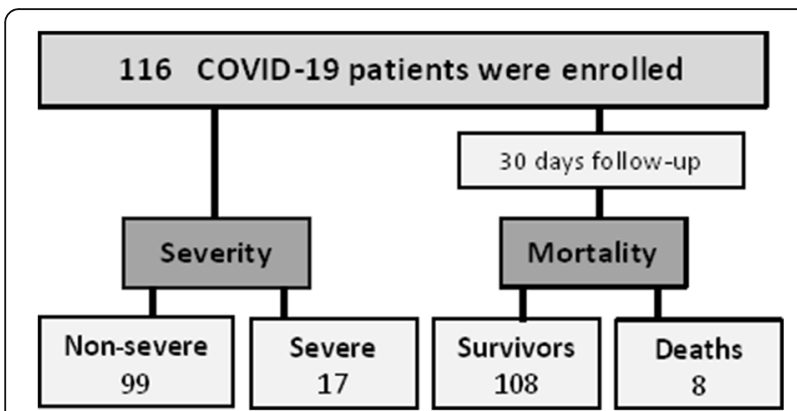

Fig. 1 Study flowchart

\section{Results}

A total of 116 COVID-19 patients were included in this study. Patients were assigned to the severe $(14.7 \%)$ and non-severe $(85.3 \%)$ groups. The demographic and clinical characteristics of patients according to severity were presented in Table 1 . The severe group had significantly higher values of patients' age and smoking percent than the non-severe group. The most prevalent symptoms were fever, cough, fatigue, and bone ache. The severe patients had a higher prevalence of dyspnea and chest tightness than that of non-severe patients. Severe patients were more likely to have co-morbidities in comparison with non-severe patients. In this cohort, eight patients (6.9\%) died in the hospital. No mortality was detected among those with the non-severe disease.

The laboratory findings of the studied patients at the time of diagnosis are presented in Table 2; the patients were sub-grouped according to the COVID-19 severity. Severe patients had higher levels of IL-6, CRP, and CRP/ albumin ratio. On the other hand, the levels of albumin were decreased in severe ones.

The role of laboratory tests in discriminating the severity of COVID-19 was evaluated. The ROC curve analysis was utilized. Non-severe patients were considered negative and severe patients as positive. The area under the ROC curve (ROC-AUC) was assessed (Fig. 2). IL-6 is the most sensitive predictor of COVID-19 severity. The performance criteria of the laboratory markers were presented in Table 2.

Further evaluation of the association between IL- 6 and COVID-19 severity was performed by Logistic Regression Analysis (Table 3). IL-6 had OR of 87.7 (95\% CI = 18.9-408.2) $(P<0.0001)$. Three models of adjustment were proposed. Model-1 adjustment utilizes the other significant laboratory tests includes CRP, CRP- albumin ratio, and albumin. In model 2, IL-6 had an adjusted OR of 79.1 ( $95 \% \mathrm{CI}=10.6-588.1)$. Model 2 was the adjustment for demographic and clinical data includes age, sex, smoking, hypertension, diabetes, chest diseases, thyroid diseases, cardiac diseases, dyspnea, chest tightness, and ansomia. IL-6 had an adjusted OR of 38.9 (95\% CI =
2.6-589). Model-3 adjustment included all variables used in model 1 and model 2, and IL-6 had an adjusted OR of $30.8(95 \% \mathrm{CI}=1.1-728.3)(P=0.046)$. So, $\mathrm{IL}-6$ can be an independent predictor of COVID-19 severity.

During the follow-up period, eight patients died (6.9\%). The follow-up period for the mortality data was varied between 1 and 30 days, with a median of 14 days. The overall survival of all patients was $93.1 \%$. Regarding patient's age, non-survivors had significant higher values than survivor (63.5 [31-88] and 37 [20-78] respectively; $P=0.003)$. Also, the prevalence of co-morbidities in non-survivors was significantly higher than survivors (87.5\% and $31.5 \%$, respectively; $P=0.001$ ).

The importance of laboratory tests in detecting the COVID-19 mortality was assessed by ROC curve analysis. The laboratory tests had the highest ROC-AUC were CRP/albumin ratio, CRP, and IL-6. The ROC-AUC values were $0.955,0.939$, and 0.923 , respectively. CRP/albumin ratio is the sensitive predictor of COVID-19 mortality (Fig. 3a). At CRP/albumin ratio value of 11.4 (the highest Youden's index $=0.8$ ), $\mathrm{CRP} /$ albumin ratio can predict mortality outcome. The COVID-19 patients were divided into two groups according to their serum CRP/ albumin ratio values levels (low ratio group $\leq 11.4$ and high ratio group $>11.4$ ). The 30-day mortality rates were $0.99 \%$ and $46.7 \%$ in a group of low ratios and high ones, respectively. The overall survival was assessed by the Kaplan-Meier curve (Fig. 3b), it showed lower survival in patients with elevated CRP/albumin ratios (log-rank test, $P<0.0001)$. The Cox Regression Analysis showed that a high CRP/albumin ratio of $>11.4$ was associated with COVID-19 mortality (hazard ratio $=59.9,95 \% \mathrm{CI}=$ 7.4-488.3, and $P<0.0001$ ). High CRP/albumin ratio had an adjusted hazard ratio of $26.5(95 \% \mathrm{CI}=2.6-270.7, P=$ 0.006 ) after adjustment of age and presence of comorbidities. So, the CRP/albumin ratio could be a significant independent prognostic factor for COVID-19 mortality.

\section{Discussion}

This study provided the baseline demographic, clinical, and laboratory characteristics of non-severe and severe COVID-19 patients. About 14.7\% of the studied cohort fulfills the criteria of severe cases. The percentage of severe cases was comparable to the published risk of severity percentage [12]. The current study found that severe and non-survivor patients were elderly. The age of the patients is associated with worse disease progress and a higher mortality rate [13]. The smoking percentage was higher in severe cases. In the same line, Guo [14] revealed by a meta-analysis that smoking was associated with COVID-19 severity. Co-morbidities including hypertension, diabetes, cardiovascular, renal, hepatic, and chest diseases were prevalent in severe and 
Table 1 Demographic, clinical, and laboratory characteristics of the patients

\begin{tabular}{|c|c|c|c|}
\hline Parameters & Non-severe (no.: 99) & Severe (no.: 17) & $P$ \\
\hline Age, years & $36[21-77]$ & $54.5[20-88]$ & $0.001^{*}$ \\
\hline Sex, male & $51(51.5)$ & $12(70.6)$ & 0.15 \\
\hline Smoking & $6(6.1)$ & $4(23.5)$ & $0.018^{*}$ \\
\hline \multicolumn{4}{|l|}{ Symptoms } \\
\hline - Fever & $85(85.9)$ & $16(94.1)$ & 0.35 \\
\hline - Fatigue & $22(22.2)$ & $5(29.4)$ & 0.52 \\
\hline - Bone ache & $17(17.2)$ & $4(23.5)$ & 0.53 \\
\hline - Anosmia & $55(55.6)$ & $4(23.5)$ & $0.015^{*}$ \\
\hline - Nausea or vomiting & $3(3)$ & $1(5.9)$ & 0.55 \\
\hline - Diarrhea & $5(5.1)$ & $2(11.8)$ & 0.28 \\
\hline - Abdominal pain & $9(9.1)$ & $3(17.6)$ & 0.29 \\
\hline - Sore throat & $9(9.1)$ & $3(17.6)$ & 0.29 \\
\hline - Cough & $72(72.7)$ & $12(70.6)$ & 0.86 \\
\hline - Dyspnea & $4(4)$ & $10(58.8)$ & $<0.0001^{*}$ \\
\hline - Chest tightness & $5(5.1)$ & $7(41.7)$ & $<0.0001^{*}$ \\
\hline \multicolumn{4}{|l|}{ Co-morbidities } \\
\hline - Diabetes & $8(8.1)$ & $5(29.4)$ & $0.01^{*}$ \\
\hline - Hypertension & $12(12.1)$ & $6(35.3)$ & $0.015^{*}$ \\
\hline - Coronary heart disease & $2(2)$ & $3(17.6)$ & $0.003^{*}$ \\
\hline - Chest diseases & $5(5.1)$ & $4(23.5)$ & $0.009^{*}$ \\
\hline - Thyroid diseases & $0(0)$ & $1(5.9)$ & $0.015^{*}$ \\
\hline \multicolumn{4}{|l|}{ Vital signs } \\
\hline - Respiratory rate, breaths/min & $22[20-23]$ & 23 [21-30] & $<0.0001^{*}$ \\
\hline - Oxygen saturation, \% & 95 [94-97] & 87 [72-94] & $<0.0001^{*}$ \\
\hline \multicolumn{4}{|l|}{ Outcome } \\
\hline - Mortality & $0(0)$ & $8(47.1)$ & $<0.0001^{*}$ \\
\hline \multicolumn{4}{|l|}{ Laboratory parameters } \\
\hline • $\mid \mathrm{L}-6, \mathrm{pg} / \mathrm{mL}$ & $14.5[6.8-47.5]$ & $55.9[20.5-123.5]$ & $<0.0001^{*}$ \\
\hline - CRP, mg/L & $20.5[6-73]$ & 46 [18.5-183] & $<0.0001^{*}$ \\
\hline - Albumin, g/dL & $4[3-5.3]$ & $3.5[2.8-5]$ & $0.024^{*}$ \\
\hline - CRP/albumin ratio & $5.1[1.6-18.7]$ & $14.1[5.8-48.2]$ & $<0.0001^{*}$ \\
\hline
\end{tabular}

Data are expressed as median [range] or number (\%)

"Significant

Table 2 The performance criteria of the laboratory markers in discriminating the severity of COVID-19

\begin{tabular}{|c|c|c|c|c|c|c|c|c|}
\hline Parameters & Cutoff & Youden's index & AUC $(95 \% \mathrm{Cl})$ & Sensitivity & Specificity & PPV & NPP & Accuracy \\
\hline IL-6, pg/mL & $>32.3$ & 0.77 & $0.955(0.912-0.997)$ & $82.4 \%$ & $94.4 \%$ & $73.7 \%$ & $96.9 \%$ & $93.1 \%$ \\
\hline CRP/albumin ratio & $>8.9$ & 0.73 & $0.922(0.862-0.981)$ & $82.4 \%$ & $90.9 \%$ & $60.9 \%$ & $96.8 \%$ & $89.7 \%$ \\
\hline CRP, mg/L & $>33.9$ & 0.65 & 0.889 (0.805-0.973) & $76.5 \%$ & $88.9 \%$ & $54.2 \%$ & $95.7 \%$ & $87.1 \%$ \\
\hline Albumin, g/dL & $<3.8$ & 0.42 & $0.672(0.537-0.806)$ & $82.4 \%$ & $60.6 \%$ & $26.4 \%$ & $95.2 \%$ & $63.8 \%$ \\
\hline
\end{tabular}

AUC area under the ROC; Cl confidence interval; CRP C-reactive protein; IL-6 interleukin 6 


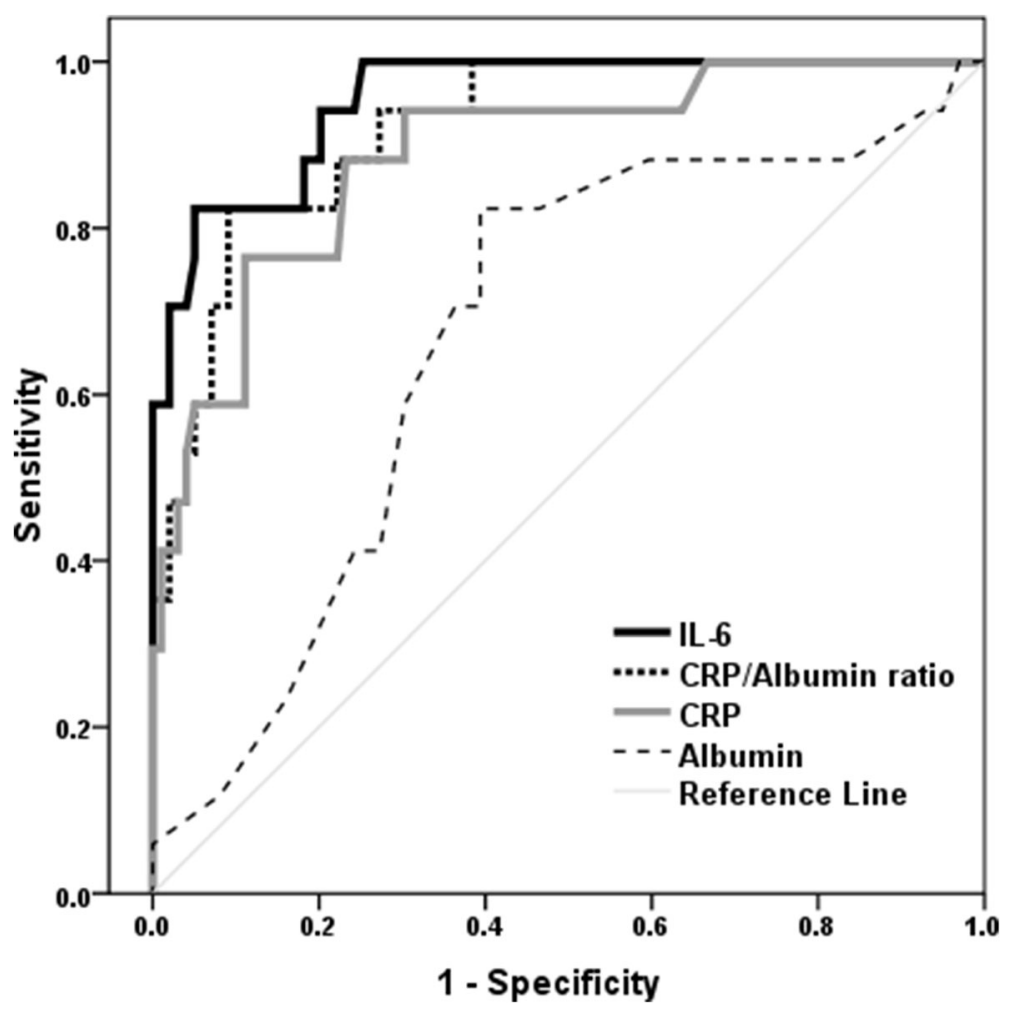

Fig. 2 ROC curve of laboratory markers as predictors of COVID-19 severity

non-survivor cases. This finding is in agreement with that of Yang et al. [15].

In this study, the most prevalent symptoms were fever, cough, fatigue, and bone ache. Dyspnea, chest tightness, and anosmia were the clinically significant discriminator between the non-severe and severe groups. The main symptom among COVID-19 cases was fever. Fever was presented in nearly eighty percent of the cases [16]. The second prevalent symptom was coughing [17]. The severe patient had a higher prevalence of dyspnea. Dyspnea seems to be a marker of severity [18]. Other detected symptoms include headache, fatigue, sore

Table 3 IL-6 as a predictor of COVID-19 severity

\begin{tabular}{llll}
\hline IL-6 & Odds ratio & 95\% Confidence interval & $\boldsymbol{P}$ \\
\hline Crude & 87.7 & $18.9-408.2$ & $<0.0001^{*}$ \\
Adjusted & & & \\
- Model 1 & 79.1 & $10.6-588$ & $<0.0001^{*}$ \\
- Model 2 & 38.9 & $2.6-589$ & $0.008^{*}$ \\
- Model 3 & 27.9 & $1.1-728.3$ & $0.046^{*}$
\end{tabular}

Model 1, IL-6 adjusted to CRP, CRP/albumin ratio, and albumin Model 2, IL-6 adjusted to age, sex, smoking, hypertension, diabetes, chest diseases, thyroid diseases, cardiac diseases, dyspnea, chest tightness, and anosmia

Model 3, IL-6 adjusted to all parameters included in model 1 and model 2 "Significant throat, and gastrointestinal symptoms were varying in prevalence [19].

The mortality rate in 30-day follow up was $6.9 \%$. High mortality was significant in older age cases and the presence of co-morbidities. This mortality rate does not reflect the real population mortality rate due to the absence of population screening that underestimates the total number of patients [20].

This study aimed to evaluate the role of laboratory markers at the time of diagnosis in estimating the severity and predicting the prognosis of COVID-19. As far as the researcher knows, this study is the first to evaluate the role of CRP/albumin ratio in COVID-19 mortality prediction. This study showed that severe patients had significantly higher values of CRP, CRP/albumin ratio, and IL-6. On the other hand, the albumin levels decreased in the severe ones. ROC curve analysis revealed that the best discriminator of disease severity was IL- 6 while CRP/albumin ratio was the best predictor of adverse outcome.

CRP is considered a sensitive biomarker of infection, inflammation, and tissue damage. During the acute inflammatory responses, the CRP level increases rapidly [21]. CRP suggested as an efficient marker in assessing COVID-19 severity [22]. This study revealed that CRP was associated with disease severity and clinical outcome. But IL-6 and CRP/albumin ratio performed better 

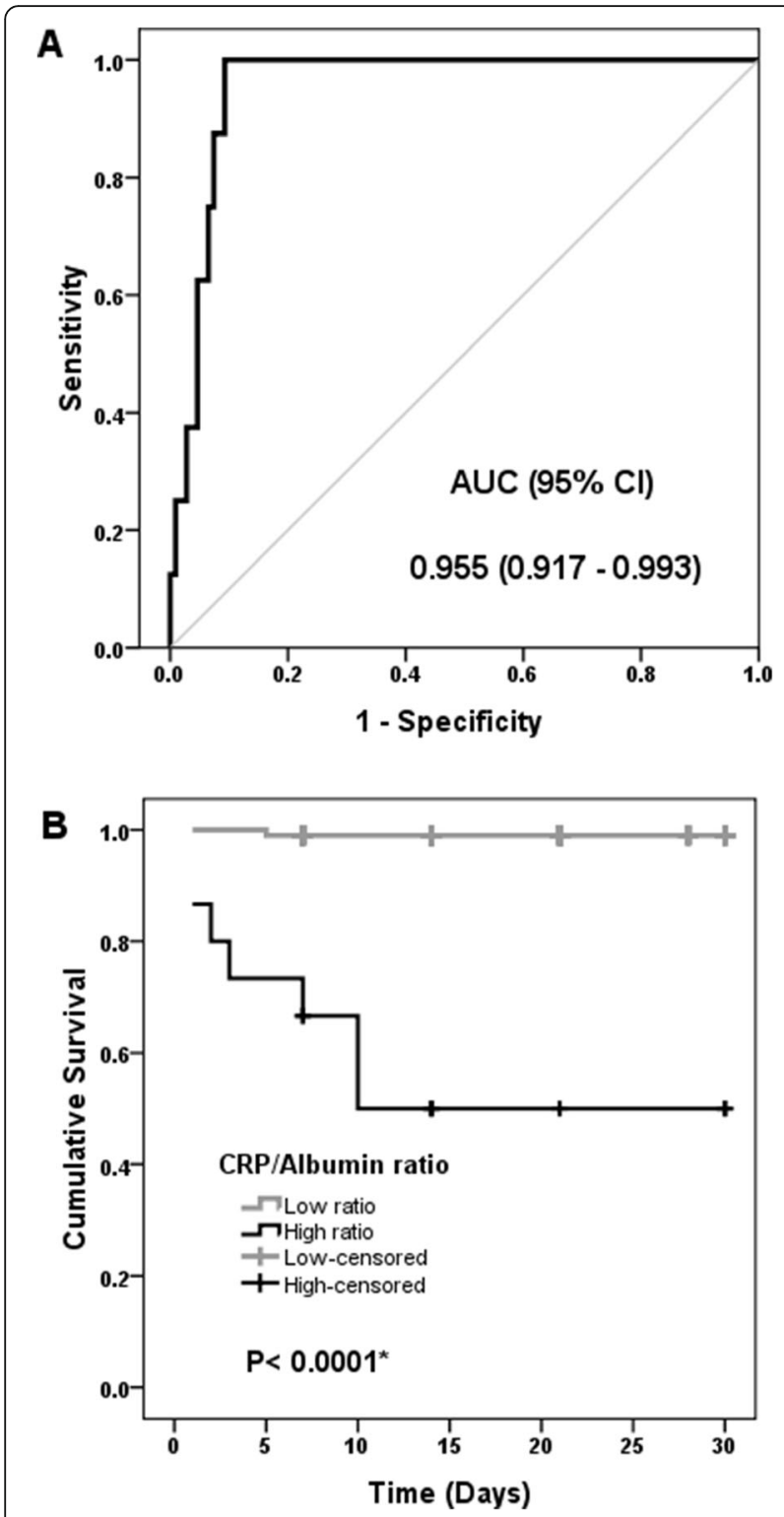

Fig. 3 a ROC curve of CRP/albumin ratio as a predictor of COVID-19 mortality. b Kaplan-Meier survival curve according to the CRP/ albumin ratio values

than CRP in predicting disease severity and adverse outcome.

The inflammatory reaction plays a prominent role in the pathophysiology of COVID-19. The proinflammatory cytokine increased in the peripheral blood of the patients [23]. The National Health Commission of China (trial 7th version) approves that peripheral blood IL-6 level increased during COVID-19 infection [24]. IL6 was defined as an acute-phase inflammatory cytokine; its serum level reflects the degree of lung inflammation [25]. The current study assessed the IL-6 levels in COVID-19 patients. The patients with severe COVID-19 had higher levels of IL-6 in comparison with non-severe patients. Regards IL-6 diagnostic performance criteria, IL-6 seems to be a useful marker for early recognition of severe disease. IL-6 showed significantly predictive power even after adjustment to different models that include clinical and laboratory significant parameters. So, IL-6 can be considered as an independent predictor of COVID-19 severity. A meta-analysis was performed on nine studies to evaluate the role of IL- 6 in assessing the COVID-19 severity. A significantly higher serum IL-6 levels were confirmed in patients with severe COVID-19 in comparison to non-severe ones [26]. These findings support that viral infection mediates lung injury via cytokines effects [27].

In this study, CRP levels alone can predict the mortality in patients with COVID-19. Significant hypoalbuminemia is detected in non-survivors COVID-19 patients. Reduced serum albumin level was associated with an increased mortality rate [28]. The CRP/albumin ratio was studied previously as a prognostic marker in critically ill patients with infections and malignancy [29]. This study showed lower survival in patients had high CRP/albumin ratios. However, albumin and CRP levels alternation may be due to the associated chronic illness [30]. Cox Regression Analysis showed that a high CRP/albumin ratio was associated with COVID-19 mortality even after adjustment to age and presence of co-morbidities. So, the CRP/albumin ratio could be a significant independent prognostic factor for COVID-19 mortality.

The current study had some limitations. First, this study was a single-center study, so further studies are required to include multiple centers with a much number of participants. Second, this study design was a cohort that lacks a control group. Lastly, the changes in the levels of the markers over time were not part of this study design, so further studies are recommended to assess their dynamic change. Future studies are suggested to confirm this study's results.

\section{Conclusion}

IL-6 level could effectively discriminate COVID-19 severity. CRP/albumin ratio was an independent risk factor for 30-day mortality rate in patients with COVID-19. IL6 and CRP/albumin ratio seem to be valuable biomarkers in evaluating the severity and prognosis of COVID-19, respectively. High levels of the biomarkers require more attention that will enable better management.

\section{Abbreviations}

Cl: Confidence interval; COVID-19: Coronavirus disease 2019; CRP: C-reactive protein; IL: Interleukin; OR: Odds ratio; ROC: Receiver operating characteristic curve; ROC-AUC: Area under the ROC curve

\section{Acknowledgements}

Not applicable 


\section{Authors' contributions}

MAE (El-Shabrawy): Conception, data acquisition, manuscript review, supervision. MEA: Design of the work, data acquisition, analysis, interpretation of data. MAE (El-Shafei): Sample analysis, data analysis, manuscript preparation. AAA: Design of the work, data acquisition, analysis, interpretation of data. ASA: Sample analysis, methodology. M M E: Conception, sample analysis, interpretation of data, data analysis, manuscript preparation. MAS: Conception, sample analysis, interpretation of data, manuscript review, supervision. All authors have read and approved the manuscript.

\section{Funding}

Not applicable

\section{Availability of data and materials}

All the data of the current study are available from the corresponding author upon reasonable request.

\section{Ethics approval and consent to participate}

Institutional Review Board of the Faculty of Human Medicine, Zagazig University (approval number: 6250). The subjects' participation was confirmed by signing the consent form either by the patient or the first degree relatives.

\section{Consent for publication}

Not applicable

\section{Competing interests}

The authors declare that they have no competing interests.

\section{Author details}

'Chest Medicine Department, Faculty of Human Medicine, Zagazig University, Zagazig, Egypt. ${ }^{2}$ Medical Microbiology and Immunology Department, Faculty of Human Medicine, Zagazig University, Zagazig, Egypt. ${ }^{3}$ Tropical Medicine Department, Faculty of Human Medicine, Zagazig University, Zagazig, Egypt. ${ }^{4}$ Histology and Histochemistry, Faculty of Science, Helwan University, Helwan, Egypt. ${ }^{5}$ Clinical Pathology Department, Faculty of Human Medicine, Zagazig University, Zagazig, Egypt.

Received: 18 September 2020 Accepted: 2 January 2021

Published online: 14 January 2021

\section{References}

1. Luk HKH, Li X, Fung J, Lau SKP, Woo PCY (2019) Molecular epidemiology, evolution and phylogeny of SARS coronavirus. Infect Genet Evol 71:21-30

2. Balkhair AA (2020) COVID-19 pandemic: a new chapter in the history of infectious diseases. Oman Med J 35(2):e123

3. Saba Al, Elsheikh AH (2020) Forecasting the prevalence of COVID-19 outbreak in Egypt using nonlinear autoregressive artificial neural networks. Process Saf Environ Prot 141:1-8

4. Singhal T (2020) A review of coronavirus disease-2019 (COVID-19). Indian J Pediatr 87(4):281-286

5. Tian S, Hu N, Lou J, Chen K, Kang X, Xiang Z et al (2020) Characteristics of COVID-19 infection in Beijing. J Inf Secur 80(4):401-406

6. Channappanavar R, Perlman S (2017) Pathogenic human coronavirus infections: causes and consequences of cytokine storm and immunopathology. Semin Immunopathol 39(5):529-539

7. Jesenak M, Brndiarova M, Urbancikova I, Rennerova Z, Vojtkova J, Bobcakova A et al (2020) Immune parameters and COVID-19 infection associations with clinical severity and disease prognosis. Front Cell Infect Microbiol 10:364

8. Aziz M, Fatima R, Lee-Smith W, Assaly R (2020) The association of low serum albumin level with severe COVID-19: a systematic review and meta-analysis. Crit Care 24:255

9. Akirov A, Masri-Iraqi H, Atamna A, Shimon I (2017) Low albumin levels are associated with mortality risk in hospitalized patients. Am J Med 130(12):1465

10. Gong J, Ou J, Qiu X, Jie Y, Chen Y, Yuan L et al (2020) A tool to early predict severe corona virus disease 2019 (COVID-19): a multicenter study using the risk nomogram in Wuhan and Guangdong, China. Clin Infect Dis 71(15):833-840
11. Liu F, Li L, Xu M, Wu J, Luo D, Zhu Y et al (2020) Prognostic value of interleukin-6, C-reactive protein, and procalcitonin in patients with COVID19. J Clin Virol 127:104370

12. Hu Y, Sun J, Dai Z, Deng H, Li X, Huang Q et al (2020) Prevalence and severity of corona virus disease 2019 (COVID-19): a systematic review and meta-analysis. J Clin Virol 127:104371

13. Wang L, He W, Yu X, Hu D, Bao M, Liu H et al (2020) Coronavirus disease 2019 in elderly patients: characteristics and prognostic factors based on 4 week follow-up. J Inf Secur 80(6):639-645

14. Guo FR (2020) Active smoking is associated with severity of coronavirus disease 2019 (COVID-19): an update of a meta-analysis. Tob Induc Dis 18:37

15. Yang J, Zheng Y, Gou X, Pu K, Chen Z, Guo Q et al (2020) Prevalence of comorbidities and its effects in patients infected with SARS-CoV-2: a systematic review and meta-analysis. Int J Infect Dis 94:91-95

16. Feng Y, Ling Y, Bai T, Xie Y, Huang J, Li J et al (2020) COVID-19 with different severities: a multicenter study of clinical features. Am J Respir Crit Care Med 201(11):1380-1388

17. Fu L, Wang B, Yuan T, Chen X, Ao Y, Fitzpatrick T et al (2020) Clinical characteristics of coronavirus disease 2019 (COVID-19) in China: a systematic review and meta-analysis. J Inf Secur 80(6):656-665

18. Zhou $Y, H e ~ Y$, Yang $H, Y u H$, Wang $T$, Chen $Z$ et al (2020) Development and validation a nomogram for predicting the risk of severe COVID-19: a multicenter study in Sichuan, China. PLoS One 15(5):e0233328

19. Chen J, Qi T, Liu L, Ling Y, Qian Z, Li T et al (2020) Clinical progression of patients with COVID-19 in Shanghai, China. J Inf Secur 80(5):1-6

20. Hassany M, Abdel-Razek W, Asem N, AbdAllah M, Zaid H (2020) Estimation of COVID-19 burden in Egypt. Lancet Infect Dis 20(8):896-897

21. Sproston NR, Ashworth JJ (2018) Role of C-reactive protein at sites of inflammation and infection. Front Immunol 9:754

22. Chen W, Zheng KI, Liu S, Yan Z, Xu C, Qiao Z (2020) Plasma CRP level is positively associated with the severity of COVID-19. Ann Clin Microbiol Antimicrob 19:18

23. Ragab D, Salah Eldin H, Taeimah M, Khattab R, Salem R (2020) The COVID-19 cytokine storm; what we know so far. Front Immunol 11:1446

24. Zhao M (2020) Cytokine storm and immunomodulatory therapy in COVID19: role of chloroquine and anti-IL-6 monoclonal antibodies. Int J Antimicrob Agents 55(6):105982

25. de Brito RC, Lucena-Silva N, Torres LC, Luna CF, Correia JB, da Silva GA (2016) The balance between the serum levels of IL- 6 and IL-10 cytokines discriminates mild and severe acute pneumonia. BMC Pulm Med 16:170

26. Aziz M, Fatima R, Assaly R (2020) Elevated interleukin-6 and severe COVID19: a meta-analysis. J Med Virol. https://doi.org/10.1002/jmv.25948

27. Liu T, Zhang J, Yang Y, Ma H, Li Z, Zhang J et al (2020) The role of interleukin-6 in monitoring severe case of coronavirus disease 2019. EMBO Mol Med 12(7):e12421

28. Huang J, Cheng A, Kumar R, Fang Y, Chen G, Zhu Y et al (2020) Hypoalbuminemia predicts the outcome of COVID-19 independent of age and co-morbidity. J Med Virol. https://doi.org/10.1002/jmv.26003

29. Park JE, Chung KS, Song JH, Kim SY, Kim EY, Jung JY et al (2018) The Creactive protein/albumin ratio as a predictor of mortality in critically ill patients. J Clin Med 7(10):333

30. Hanafusa N, Nitta K, Okazaki M, Komatsu M, Shiohira S, Kawaguchi H et al (2017) Serum albumin level adjusted with C-reactive protein predicts hemodialysis patient survival. Ren Replace Ther 3:9

\section{Publisher's Note}

Springer Nature remains neutral with regard to jurisdictional claims in published maps and institutional affiliations. 